\title{
Study on Simulation Performance of OFDM-IDMA System
}

\author{
Zhen-hua WANG ${ }^{1}$, Ge-fei $\mathrm{YU}^{1}$, Xi CHENG ${ }^{1}$, Jie Yang ${ }^{1}$, Yin-ting WANG ${ }^{2}$ \\ 1.Chongqing Communication Institute, 2.Communication Training Base of General Staff \\ Chongqing, CHINA \\ Email:cqzhenhua79@163.com; yugefei2003@yahoo.com.cn
}

\begin{abstract}
OFDM-IDMA system with perfect performance is the key technology of 4th mobile communication. in this paper, we simulated the performance of OFDM-IDMA in AWGN and quasi-static Rayleigh channel respectively, the simulation results indicate that the performance of multi-user is close to the single use as the increase of SNR, and the error-bits curve descends obviously, which means OFDM-IDMA system can be used to the complex electromagnetic environment and highspeed data transmission.
\end{abstract}

Keywords-orthogonal frequency division multiplexing (OFDM ) ; interleave division multiple access (IDMA); multi-user detection

\section{INTRODUCTION}

Today people pay more attention to the $4 \mathrm{G}$ wireless communication system because it can meet the need of more and more users to high rate and high reliability, practice shows that the integration of CDMA and OFDM can improve the performance of system greatly, it is usually thought as the key technology of 4G wireless communication system. But OFDM-CDMA system needs orthogonal codes to differentiate users, which causes two problems:

One is that the orthogonality of different users will be destroyed (especially in the uplink ) and causes serious MAI(multi-address interference) problems. In the past, it can be removed by using Multi-User Detection(MUD), but MUD is too complicated to use. For example, the complexity of Maximum a posterior (MAP) probability has exponential relationship with user numbers $K$, for the traditional MMSE, the complexity of MMSE and decorrelator is usually $2 \mathrm{~K}$, and the overhead will be increased when the $\mathrm{K}$ is larger.

On the other hand, according to the coding theory, it is not a good means to using spreading codes to differentiate users, because it can't get coding gain but result in the increase of band.

Interleave division multiple access ( IDMA ) [13]system can use interleavers to differentiate users; it exhibits excellent BER performance, good spectrum usability and low complexity of receiver. Some scholars consider it can replace CDMA of OFDM-CDMA system [4] [5]. OFDM-IDMA system inherits so many advantages of OFDM-CDMA that it can effectively deal with ISI and reduce interference between cells. The output of IDMA is very high because it uses one simple and effective Turbo iterative for MUD algorithm, on the other hand, OFDM-
IDMA doesn't need spectrum spreading because its users are differentiated by interleavers, so the whole band spreading of system can be used for FEC to get higher gain[6].

\section{OFDM SYSTEM}

OFDM system is shown as Fig.1. in the transmitter end, the input serial binary bits are first sent to the encoder and mapped to a series of symbols, and then the mapped symbols are turned into parallel signal by serial-to-parallel conversion and sent to the IFFT module to be transformed into time domain signals, the Cyclic Prefix (CP) is added before the time domain signals to deal with interference between symbols, then the output signals are converted by parallel-to-serial and D/A conversion to be sent to the wireless channel. In the receiving end, the receiving signals are first synchronized and handled by A/D conversion, then the serial signals are turned into parallel signals, CP is removed, and are sent to the FFT module, the output frequency domain signals of FFT are converted by parallelto-serial conversion and. The system can detect transmission signals by equalizing estimated channel parameters, and then the signals are sent to the de-mapper and decoder to recover binary sending sequence.

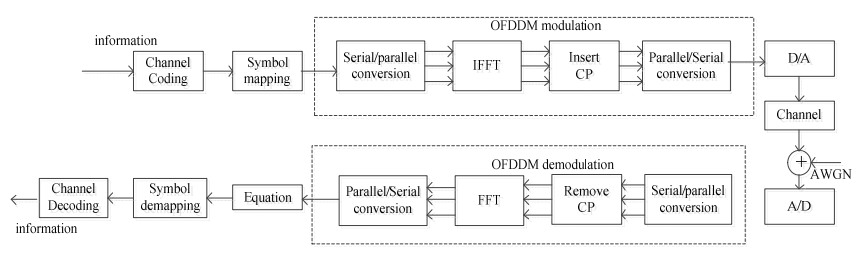

Fig.1 OFDM System

\section{IDMA SYSTEM}

The IDMA system is shown as Fig.2, in this structure, the whole band of the CDMA system is released for channel coding [10], so IDMA is usually seen as one special form of CDMA, it inherits so many advantages, such as antiinterference and anti-multipath fading performance, on the other hand, and it uses one simple chip-to-chip iterative MUD receiving structure, it has higher spectrum utilization, better communication performance and lower receiver complexity.

In the Fig.2,it is supposed there are $\mathrm{K}$ users in the sending end, the input code sequence of kth user is 
$\mathbf{d}_{k}=\left[d_{k}(1), \ldots, d_{k}(i), \ldots, d_{k}(I)\right]^{T}$ (I is the length of the information codes), $d_{k}$ is then sent to the FEC encoder $C_{F E C}$ and output $\mathbf{b}_{k}=\left[b_{k}(1), \ldots, b_{k}(l), \ldots, b_{k}(L)\right]$, its spectrum is spread in the repeater coder $C_{R E P}$, each symbol $b_{k}(l)$ is repeated $S$ times and output low rate code sequence $c_{k}=\left[c_{k}(1), \ldots, c_{k}(j), \ldots, c_{k}(J)\right]^{T}(J=L \times S$ is the length of the sending code sequence), encoders $\mathrm{C}_{F E C}$ and $\mathrm{C}_{R E P}$ constitute one low rate encoder $C$, finally, $C_{k}$ is then confused by interleaver $\left\{\pi_{k}\right\}$ and rearranged as transmission code sequence $\mathbf{x}_{k}=\left[x_{k}(1), \ldots, x_{k}(j), \ldots, x_{k}(J)\right]^{T}$, according to the regulation of CDMA system, the information bits in the $x_{k}(j)$ is usually called as "chips".

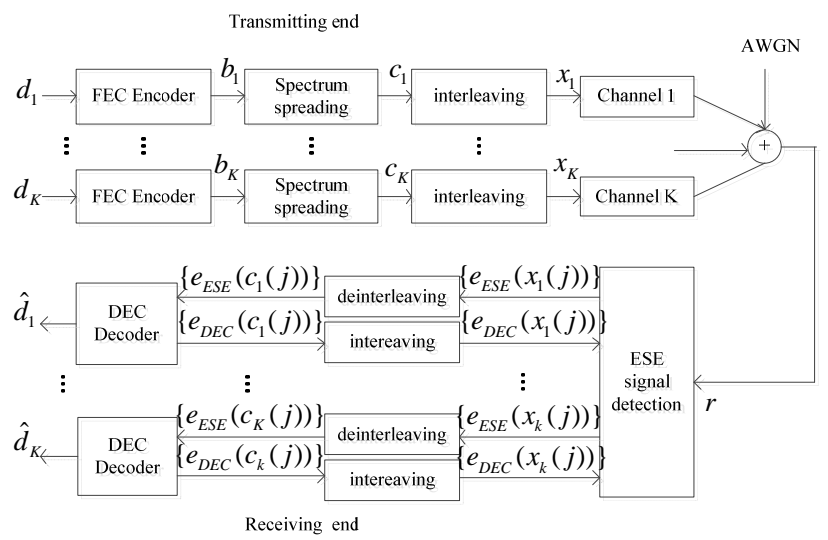

Fig.2 Transmitting and receiving scheme of IDMA system

In the receiving end, a second-optimal turbo iterative decoder consists of one signal detector and $\mathrm{k}$ APP decoders (DECs) is being used. The problems of multiple access and encoding limits can be figured out by ESE and DECs, the output of ESE and DECs is LLRs about $\left\{x_{k}(j)\right\}$, that is we usually called extra information:

$$
e\left(x_{k}(j)\right) \equiv \log \left\{\frac{\operatorname{Pr}\left(x_{k}(j)=+1\right)}{\operatorname{Pr}\left(x_{k}(j)=-1\right)}\right\}
$$

The extra information totally does one Turbo iterative receiving process from chip to chip, and after $\mathrm{M}$ time's iteration, DECs of $\mathrm{K}$ users will respectively output $\mathrm{k}$ relevant code sequence $\mathbf{d}_{k}$ and its hard decision value is $\hat{\mathbf{d}}$.

\section{OFDM-IDMA SYSTEM}

The structure of the OFDM-IDMA system is shown as Fig.3, it is supposed that $\mathbf{d}_{k}$ is the output of the kth user, $\mathbf{d}_{k}$ is sent to the FEC and RA encoders and output the sequence $C_{k}, C_{k}$ is interleaved by interleaver and mapped to the sequence $\mathbf{x}_{k}=\left[x_{k}(1), \ldots, x_{k}(j), \ldots, x_{k}(J)\right]^{T} \quad(J$ is the length of the frame), then IEFT modulates this sequences to the different carriers.

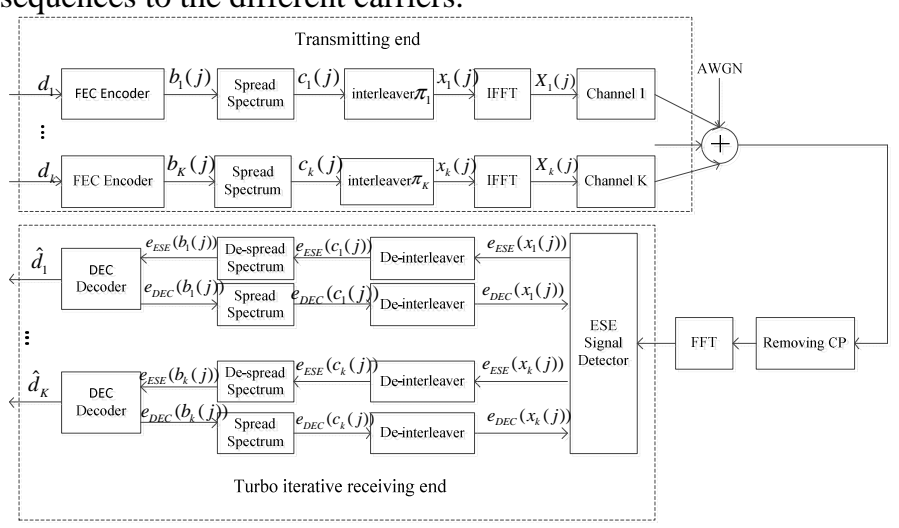

Fig.3 transmitting and receiving structure of OFDM-IDMA system

In the receiving end, OFDM signal is demodulated before iterative multi-users detection, we suppose that cyclic prefix (CP) is larger than the max delay of multi-path channels, if CP and OFDM demodulation are removed, the received signal is :

$$
r(j)=\sum_{k} H_{k}(j) x_{k}(j)+Z(j)
$$

$Z(j)$ is the transformation of complex Gauss white noise, i.e. $Z(j)=F F T(z(j)) ;\left\{H_{k}(j)\right\}$ is the fading coefficient of jth carrier.

For the OFDM-IDMA system, the fading factor $\left\{H_{k}(j)\right\}$ of OFDM sub-carrier is the same for the different $j$.

\section{PERformance ANALYsis OF OFDM-IDMA}

\section{A. Simulation Parameter}

In this simulation, the maximum iterative times of Turbo iterative receiver is 5 , the users $\mathrm{K}$ is chosen as 1,5 and 8 in the AWGN channel, BPSK modulation is used; in the multipath channel, the users $\mathrm{K}$ is chosen as 1,5,8,and 16, OPSK is used for anti-interference. All the users use the same spectrum spreading code sequence: $s=\{+1,-1,+1,-1, \ldots\}$ with the same length 16 , the chip interleaver assigned to each user is randomly output by the system. And we don't need to think about the energy distribution of the system, the number of sub-carriers on both channels is 128 , the length of $\mathrm{CP}$ is 8. 


\section{B. Performance analyzed}

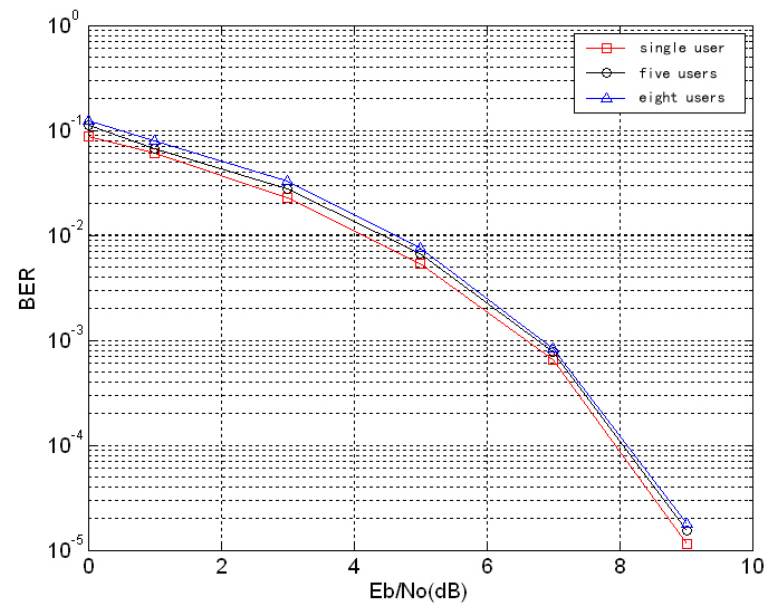

Fig.4 performance curve of OFDM-IDMA in AWGN channel

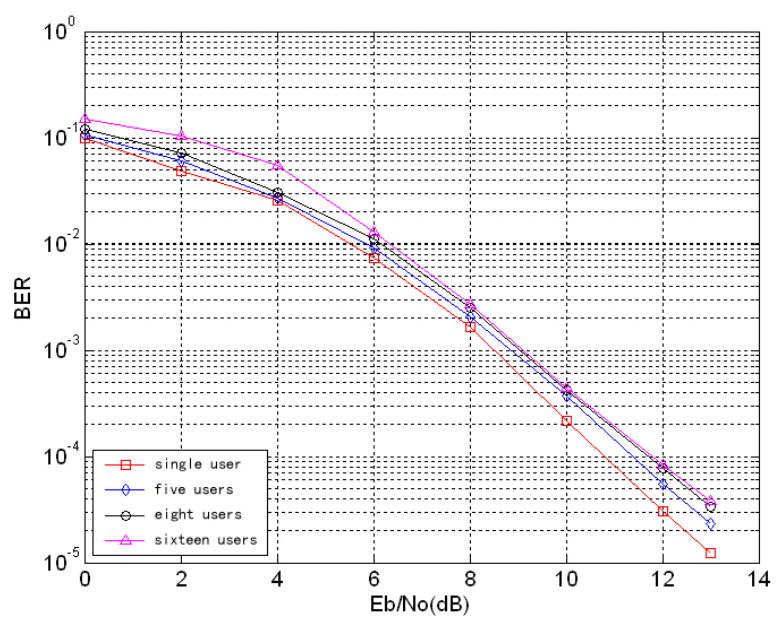

Fig.4 performance curve of OFDM-IDMA in multi- path channel

Fig.4 depicts the performance of OFDM-IDMA for multi-users in AWGN channel; it is shown that without energy distribution, the performance of system with different users is still close to the performance with one user with decreasing SNR, so the system shows the better performance for multiple users.

Fig.5 shows the BER results of OFDM-IDMA system with different users without channel coding in the quasi-static Rayleigh fading channel, the number of multiple paths is 8 . For curves are the performance of single, 5, 8 and 16 users. As shown in Fig.5, with the increasing number of users, the performance of OFDMIDMA still has the advantage in the AWGN channel; the difference between signal and multiple users is little.

\section{CONCLUSIONS}

OFDM-IDMA system structure based on theory of OFDM and IDMA is constructed in this paper, simulation results in AWGN and quasi-static Rayleigh fading channel all show that OFDM-IDMA system inherits all the advantages of OFDM and IDMA, it shows better performance than traditional multiple access technology, especially with larger users, it has more simple multi-user iterative detection algorithm and lower complexity. In a word, OFDM-IDMA based on multi-carrier modulation and interleaving multiple access technology will draw more attention in the future wireless communication.

\section{REFERENCES}

[1] LI Ping, LIU L H, WU K Y, et al. Interleave division multiple access (IDMA) communication systems[C]// Proc. 3rd International Symposium on Turbo Codes \& Related Topics, . 2003: 2157-2161.

[2] LI Ping. Interleave-division multiple access and chip-by-chip iterative multi-user detection[J]. Communications Magazine, IEEE, 2005,43(6):S19S23.

[3] LI Ping, LIU L, WU K Y, et al. Interleave-division multipleaccess[EB/OL].

[4] S. Zhou, Y. Li, M. Zhao, X. Xu, J.Wang, and Y. Yao, Novel techniques to improve downlink multiple access capacity for B3G, IEEE Commun. Mag., Jan 2005,vol. 43:61-69

[5] I. Mahafeno, C. Langlais, and C. Jego, OFDM-IDMA versus IDMA with ISIC for quasi-static Rayleigh fading multipath channels, in Proc. 4th International Symposium on Turbo Codes \& Related Topics, Munich, Germany, April 2006

[6] Kai Yang, Xiandong Wang. A Multicarrier Chip-interleaved Multiuser UWB System. ICASSP 2005, March 2005 Page(s):iii/325-iii/328 